\title{
The modulation of SiO maser polarization by Jovian planets
}

\author{
H. Wiesemeyer \\ Institut de Radioastronomie Millimétrique, 300 rue de la Piscine, Domaine Universitaire, 38406 Saint Martin d'Hères, France \\ e-mail: wiesemey@iram.fr
}

Received 17 December 2008 / Accepted 27 March 2009

\begin{abstract}
Aims. Searching for planets in the atmosphere of AGB stars is difficult, due to confusion with the stellar wind and pulsations. The aim here is to provide a complementary strategy for planet searches in such a dense environment.

Methods. The polarization properties of SiO masers, especially their circular polarization, are, under certain conditions, good tracers of rapid magnetospheric events. A Jovian planet with a magnetosphere whose dipole axis is misaligned with its rotation axis naturally provides such conditions. Here I present several models showing that the polarization will be periodically modulated.

Results. The linear and circular polarization of an $\mathrm{SiO}$ maser in a planetary magnetosphere is modulated by the precessing dipole component of the latter. The effect is measurable in saturated masers, while unsaturated masers only exhibit weak changes, because of dilution effects, and because the circular polarization there stems from the Zeeman effect making it as weak as for thermal radiation. The situation would change if anisotropic pump- and loss-rates were included, which would increase the fractional linear and, via magnetorotation, the circular polarization of the modulation.

Conclusions. Single-dish monitoring with a dense enough time sampling and a carefully calibrated polarimeter, in combination with VLBI observations, are suited to detecting and locating a periodic modulation of the circular maser polarization due to a precessing Jovian magnetosphere. The phenomenon will be rare, because a favorable arrangement of maser and magnetosphere is needed. Otherwise the polarization may be below the detection threshold, especially if the maser is unsaturated. Though exhibiting a qualitatively similar modulation, linear polarization is likely to suffer more from confusion due to dilution of the magnetosphere within the maser cross section, even in VLBI observations.
\end{abstract}

Key words. polarization - masers - stars: AGB and post-AGB - stars: magnetic fields - planetary systems - stars: atmospheres

\section{Introduction}

Most $\mathrm{SiO}$ masers are hosted by the extended atmosphere of evolved stars of low- to intermediate mass in the upper part of the asymptotic giant branch, before they evolve towards central stars of planetary nebulae (for a review see e.g., Herwig 2005). Under certain conditions, these masers trace the magnetic field by virtue of their polarization. After the first theoretical consideration of a Zeeman laser (Sargent et al. 1967), astronomical maser polarization has been extensively discussed, e.g., by Goldreich et al. (1977), Western \& Watson (1984, for linear polarization), Deguchi \& Watson (1986, for circular polarization), and Elitzur (1991). The models are compared by Gray (2003). The environment of $\mathrm{SiO}$ masers in AGB atmospheres is characterized by a dense wind driven by the stellar pulsations. Its interaction with planets engulfed in the stellar atmosphere has already been addressed by Struck-Marcell (1988) who investigated the hypothesis that $\mathrm{SiO}$ masers may form in the magnetosphere of Jovian planets. Struck et al. (2002, 2004) propose scenarios along the same lines, and model the dynamic effects of episodic accretion onto the planets. In a different context, the consequences of planets in the stellar atmosphere have been investigated by Soker (2001), who shows that Jovian and even Earth-like planets can efficiently spin-up the star after migration into the stellar atmosphere, such that it later may form an elliptical rather than a spherical planetary nebula.

Here I propose to resume the idea of Struck-Marcell (1988) that $\mathrm{SiO}$ masers (though not all) may originate in Jovian magnetospheres, leading to circular maser polarization

\footnotetext{
^ On leave to Instituto de Radioastronomía Milimétrica, Granada, Spain.
}

(Barvainis et al. 1987; Herpin et al. 2006), which may be as strong as $\sim 10 \%$ of the Stokes $I$ flux. I show that the densely sampled time series of all Stokes parameters yield a rare, but observable polarization signature characteristic of a precessing magnetosphere. Since AGB stars are slow rotators, a precession period as fast as $\sim 10 \mathrm{~h}$ hints at a planetary magnetosphere. $\mathrm{SiO}$ masers are an ideal tool for sounding Jovian planets, since they arise in a zone extending from 1.5 to $7 \mathrm{AU}$ distance from the star where they are radiatively and collisionally pumped (Gray et al. 2009), before the $\mathrm{SiO}$ molecule becomes underabundant for maser action thanks to condensation in the dust envelope. The $\mathrm{SiO}$ maser zone thus corresponds to a distance from the star where the solar system harbors its giant gas planets (Jupiter is at about $5 \mathrm{AU}$ from the sun, and Saturn at about $10 \mathrm{AU}$ ). A direct comparison between the solar sytem now and in the AGB phase is not valid, though, because planetary orbits may be dragged inwards by tidal friction, or expelled by the kinematical effect of stellar mass loss (Villaver \& Livio 2006, further references therein). Arguing with Soker (1996) that elliptical planetary nebulae formed from stars spun up by inward migration of a planet, it is assumed here that the zone in question harbors Jovian planets, since $50 \%$ of all planetary nebulae are not elliptical (Soker 2001).

The first observational evidence of extrasolar planets around evolved stars was given by Silvotti et al. (2007) who indirectly detected a planet around a post-red giant star, measuring the pulse modulations of the variability of the latter. As for the planets detected around pulsars (with a similar method, measuring the period variations of the latter) by Wolszczan \& Frail (1992), it is not yet certain whether these planet-sized bodies survive the supernova explosion, or whether they formed in the debris disk 
forming in reponse to a "fallback" (Wang et al. 2006) of ejected matter. Most extrasolar planets have so far been detected around main-sequence stars by radial velocity measurements (for a review see Santos 2008). As for the earliest phases of stellar evolution, a "hot Jupiter" has recently been discovered around the T Tauri star TW Hya (Setiawan et al. 2008). Around evolved stars, ten companions of substellar mass have been discovered to this day, the last one being the K0 giant HD17092 (Niedzielski et al. 2007, further references therein). There is no evidence yet from AGB stars, mainly because the strong, dense wind makes radial velocity measurements of the star difficult. The following models of maser polarization modulation due to precessing planetary magnetospheres are intended as a complement to exoplanet searches around AGB stars with different methods.

\section{Description of the model}

\subsection{Physical characteristics}

$\mathrm{SiO}$ is non-paramagnetic, with a Zeeman splitting of $g \Omega\left[\mathrm{s}^{-1}\right] \sim$ $10^{3} B[\mathrm{G}]$ (cf. Watson \& Wyld 2001). For a $1000 \mathrm{~K}$ gas and the magnetic fields considered here (of up to $10 \mathrm{G}$ ), the spectral linewidth is greater than the Zeeman splitting by a factor of $\sim 100$. This weak Zeeman splitting still produces a fractional circular polarization (hereafter $p_{\mathrm{C}}$ ) of $\sim 1 \%$, which is observable for a carefully designed polarimeter (e.g., Thum et al. 2008). While linear polarization may not only be caused by magnetic fields, but also by anisotropic pumping in conjunction with the Hanle effect (Bujarrabal \& Rieu 1981; Western \& Watson 1984; Asensio Ramos et al. 2005), circular polarization is a tracer of magnetic flux density as long as the Zeeman splitting $g \Omega$ is much higher than the stimulated emission rate $R$ and the loss rate $\Gamma$. As soon as $R$ is close to $g \Omega$, the circular polarization will become intensity-dependent when the maser radiation saturates (Nedoluha \& Watson 1994), thus ceasing to be a reliable tracer of magnetic fields.

To keep the model simple and to separate the predicted effects from those issued by models involving anisotropic pumpand loss-rates (see Sect. 3.3), the pump and loss events have been assumed to be isotropic here. Following Watson \& Wyld (2001), the approach of phenomenological maser theory is used. I model the polarization properties of the $v=1, J=1-0$ transition with rest frequency $\omega_{\mathrm{R}}=2.71 \times 10^{11} \mathrm{~s}^{-1}$, allowing for a straightforward analytical formulation of the problem. The rate equations yield the population differences $n_{+}, n_{0}$ and $n_{-}$between the splitup upper state $(J=1, M=+1,0,-1$, respectively) and the lower state $(J=0, M=0)$. These population differences are normalized by the ratio of the differential pump rate (into the $J=1$ respectively $J=0$ levels) to the loss rate (assumed to be the same for both levels), and are a function of molecular velocity. Because of the isotropic pumping by collisions or unpolarized radiation assumed here, the pump rates are equal into the sublevels of the $J=1$ state. Collisionally induced transitions between magnetic sublevels of a given $J$ state are neglected. This yields

$$
\begin{aligned}
& n_{+}=\frac{\left(1+R_{-}\right)\left(1+R_{0}\right)}{\left(1+2 R_{+}\right)\left(1+R_{0}\right)\left(1+R_{-}\right)+R_{0}\left(1+R_{-}\right)\left(1+R_{+}\right)+R_{-}\left(1+R_{+}\right)\left(1+R_{0}\right)}, \\
& n_{0}=\frac{\left(1+R_{+}\right)\left(1+R_{-}\right)}{\left(1+2 R_{+}\right)\left(1+R_{0}\right)\left(1+R_{-}\right)+R_{0}\left(1+R_{-}\right)\left(1+R_{+}\right)+R_{-}\left(1+R_{+}\right)\left(1+R_{0}\right)}, \\
& n_{-}=\frac{\left(1+R_{+}\right)\left(1+R_{0}\right)}{\left(1+2 R_{+}\right)\left(1+R_{0}\right)\left(1+R_{-}\right)+R_{0}\left(1+R_{-}\right)\left(1+R_{+}\right)+R_{-}\left(1+R_{+}\right)\left(1+R_{0}\right)} .
\end{aligned}
$$

Here the stimulated emission rates $R_{ \pm}$and $R_{0}$ for the transitions with $\Delta M= \pm 1$ and 0 are normalized by the saturation intensity
Table 1. Doppler factors for evaluation of the stimulated emission rates.

\begin{tabular}{cccc}
\hline \hline $\begin{array}{c}\text { Normalized stimulated } \\
\text { emission rate }\end{array}$ & \multicolumn{3}{c}{ Doppler factors $\mathcal{D}$ for evaluating } \\
$n_{+}$ & $n_{0}$ & $n_{-}$ \\
\hline$R_{+}$ & 1 & $\frac{\omega_{\mathrm{R}}+g \Omega / 2}{\omega_{\mathrm{R}}}$ & $\frac{\omega_{\mathrm{R}}+g \Omega / 2}{\omega_{\mathrm{R}}-g \Omega / 2}$ \\
$R_{0}$ & $\frac{\omega_{\mathrm{R}}+g \Omega / 2}{\omega_{\mathrm{R}}}$ & 1 & $\frac{\omega_{\mathrm{R}}}{\omega_{\mathrm{R}}-g \Omega / 2}$ \\
$R_{-}$ & $\frac{\omega_{\mathrm{R}}-g \Omega / 2}{\omega_{\mathrm{R}}+g \Omega / 2}$ & $\frac{\omega_{\mathrm{R}}-g \Omega / 2}{\omega_{\mathrm{R}}}$ & 1 \\
\hline
\end{tabular}

$I_{\mathrm{S}}=8 \hbar \omega^{3} \Gamma / 3 \pi c^{2} A$ (A being the Einstein coefficient for spontaneous emission) and are, for a given observing frequency $\omega$, evaluated at frequencies $\omega \mathcal{D}$, where $\mathcal{D}$ are the Doppler factors given in Table 1.

These Doppler factors account for the fact that molecules at different line-of-sight velocities - due to their velocity distribution and, if present, a velocity gradient (not considered here) couple to different Zeeman components. The stimulated emission rates $R_{ \pm}$and $R_{0}$ are given by (Goldreich et al. 1973)

$$
\begin{aligned}
R_{ \pm}= & I_{ \pm}\left(1+\cos ^{2} \gamma\right)+\sin ^{2} \gamma\left(Q_{ \pm} \cos 2 \eta-U_{ \pm} \sin 2 \eta\right) \\
& \pm 2 V_{ \pm} \cos \gamma \\
R_{0}= & 2 \sin ^{2} \gamma\left(I_{0}-Q_{0} \cos 2 \eta+U_{0} \sin 2 \eta\right)
\end{aligned}
$$

where $I, Q, U$ and $V$ are the Stokes parameters, normalized by the saturation intensity and multiplied ${ }^{1}$ by $4 \pi$, and the indices , \pm 0 indicate the frequency at which these quantities are to be evaluated (Table 1). Here, $\gamma$ is the angle between the magnetic field and the line-of-sight, directed outwards from the drawing plane of Fig. 1. $\eta$ is the angle between the $z$ axis and the projection of the magnetic field onto the plane of the sky (i.e., the drawing plane in Fig. 1), $B_{\text {sky }}=\sqrt{B_{y}^{2}+B_{z}^{2}}$. These angles are calculated from

$\cos \gamma=\frac{B_{x}}{\|\boldsymbol{B}\|}, \cos \eta=\frac{B_{z}}{\|\boldsymbol{B}\|}$.

For a static magnetic field, the coordinate system is conveniently chosen such that $\eta=0^{\circ}$ (i.e., the system in which Stokes $U$ vanishes in the absence of magnetorotation).

\subsection{Radiative transfer}

Since the light-travel time is negligible with respect to the time scale on which the magnetic field will vary, the stationary radiative transfer equation is used:

$\frac{\mathrm{d}}{\mathrm{d} \tau}\left(\begin{array}{c}I \\ Q \\ U \\ V\end{array}\right)=\mathbf{K}\left(\begin{array}{c}I \\ Q \\ U \\ V\end{array}\right)$

As usual in maser theory, the source term taking spontaneous emission into account is insignificant here, and $\tau$ is the opacity along the line of sight for the polarized plus unpolarized radiation. The Müller matrix $\mathbf{K}$ of the absorption coefficients has the elements

$\mathbf{K}=\left(\begin{array}{cccc}A & B & F & C \\ B & A & E & G \\ F & -E & A & D \\ C & -G & -D & A\end{array}\right)$

\footnotetext{
1 For a linear maser considered here, intensity means its solid-angle average multiplied by $4 \pi$.
} 


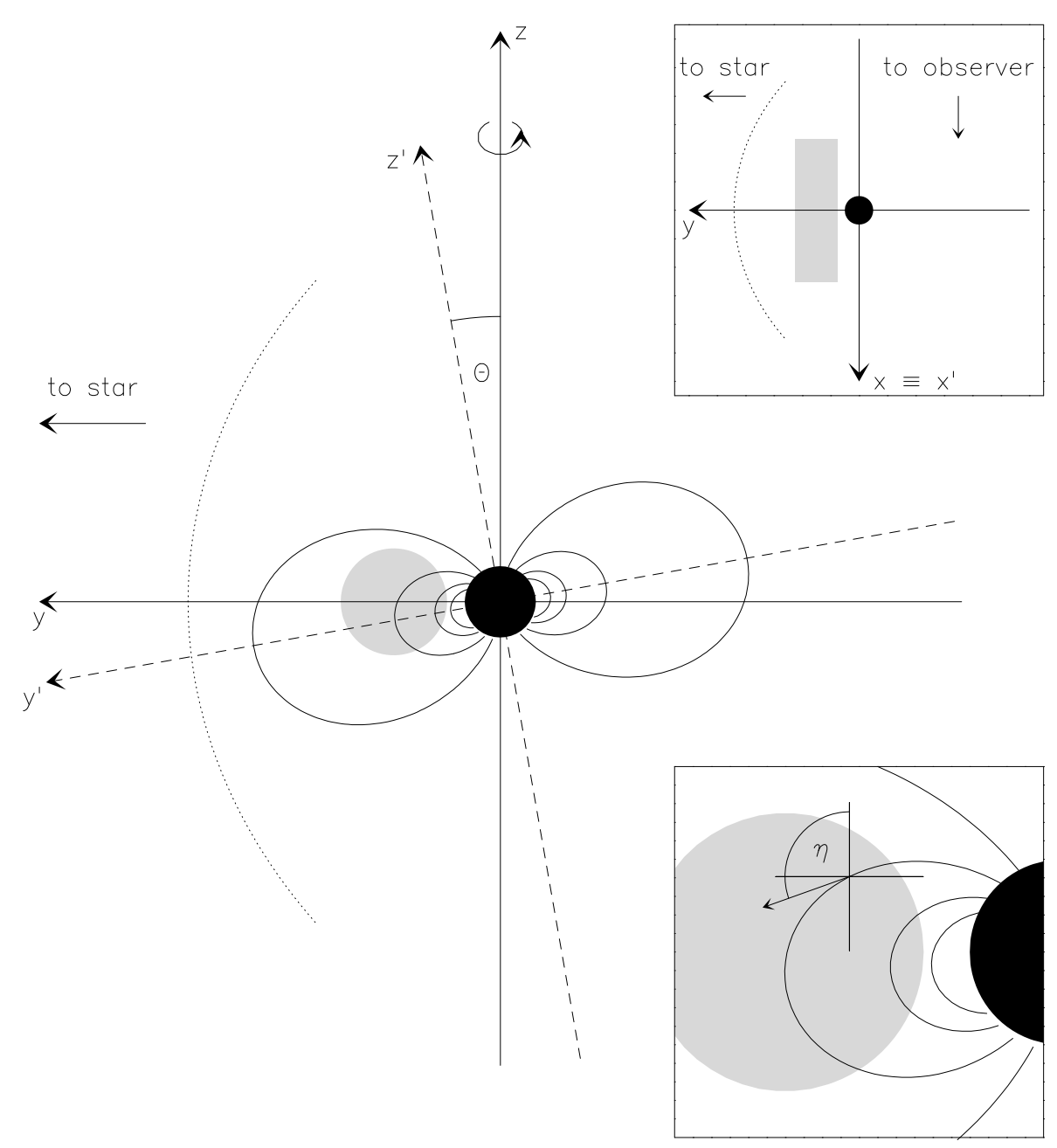

Fig. 1. Sketch of the model with magnetic field lines. The $x, y, z$ coordinate system (solid lines) is defined by the rotation axis of the planet ( $z$ axis) and the line of sight to the observer ( $x$ axis, directed from the drawing plane outwards). The coordinate system $x^{\prime}, y^{\prime}, z^{\prime}$ (dashed lines) of the magnetosphere is defined by the tilt angle $\Theta$ of the dipole axis with respect to the rotation axis. The rotation phase here is chosen such that the $x^{\prime}$ axis is identical to the $x$ axis. The cross section of the planet is depicted by the black circle, the maser slab by the grey one (for models $1 \mathrm{~b}, 2 \mathrm{~b}$ ). The location of the Chapman-Ferraro magnetopause is indicated by the dotted line. The top right insert shows the $(x, y)$ plane with the location of the magnetopause (dashed line) and the maser slab as a grey rectangle (field lines omitted). The bottom right insert shows a zoom onto a particular line of sight, marked by a cross, within the $\mathrm{SiO}$ maser bundle. The arrow indicates the local magnetic field direction and the definition of angle $\eta$.
If the vertical polarization is along the projection of the magnetic field onto the plane of the sky, $\mathbf{B}_{\text {sky }}$, the elements of $\mathbf{K}$ have the following meaning: Coefficients $A, B, C$ and $F$ are the absorption coefficients for different polarization states, while $D, E$ and $G$ describe anomalous dispersion effects (Landi degl'Innocenti \& Landi degl'Innocenti 1981). Elements $F$ and $G$ are assumed to be negligible, since $g \Omega \gg R$. This condition is necessary here, otherwise nothing about the magnetic field can be inferred from measurements of $p_{\mathrm{C}}$, since a given molecular state would be deexcited by a stimulated emission before a full Larmor precession is accomplished. The matrix elements $A, B$, and $C$ are, for a twolevel system with split-up upper level, given by (Watson \& Wyld 2001),

$A=\left(1+\cos ^{2} \gamma\right)\left(f_{+}^{(\mathrm{r})} n_{+}+f_{-}^{(\mathrm{r})} n_{-}\right)+2 f_{0}^{(\mathrm{r})} n_{0} \sin ^{2} \gamma$,

$B=\sin ^{2} \gamma\left(f_{+}^{(\mathrm{r})} n_{+}+f_{-}^{(\mathrm{r})} n_{-}-2 f_{0}^{(\mathrm{r})}\right)$,

$C=2 \cos \gamma\left(f_{+}^{(\mathrm{r})} n_{+}-f_{-}^{(\mathrm{r})} n_{-}\right)+2 f_{0}^{(\mathrm{r})} n_{0} \sin ^{2} \gamma$.

The terms $D$ and $E$ describe magneto-rotation, i.e., the conversion of linear to circular polarization, or - in other words - the generation of the latter by means other than the Zeeman effect. They are given by

$D=\sin ^{2} \gamma\left(f_{+}^{(\mathrm{i})} n_{+}+f_{-}^{(\mathrm{i})} n_{-}-2 f_{0}^{(\mathrm{i})}\right)$,

$E=2 \cos \gamma\left(f_{+}^{(\mathrm{i})} n_{+}-f_{-}^{(\mathrm{i})} n_{-}\right)+2 \frac{\mathrm{d} \eta}{\mathrm{d} \tau}$

where the extra term $2 \mathrm{~d} \eta / \mathrm{d} \tau$ in $E$ accounts for the rotation of the coordinate system, while the photon propagates along the line-of-sight, such that vertical polarization is always along $B_{\text {sky }}$. This extra term is a lengthy expression calculated from the magnetic field Eqs. (19) to (21) and the transformation defined by Eqs. (22), (23) (see below). To correctly handle phase shifts induced by magneto-rotation, the profile function $f$ is complex, where $f_{+}^{(\mathrm{r})}$ and $f_{+}^{(\mathrm{i})}$ are its real and imaginary part, respectively, at the normalized frequency offset

$v_{+}=\left(\omega-\left[\omega_{0}+g \Omega / 2\right]\right) \frac{1}{\Delta \omega_{\mathrm{D}}}$

where $\Delta \omega_{\mathrm{D}}$ is the Doppler width of the line, and $\omega_{0}$ the resonance rest frequency of the transition. Here I use $\Delta \omega_{\mathrm{D}}=$ $5.4 \times 10^{5} \mathrm{~s}^{-1}$, which corresponds to the thermal linewidth of $\mathrm{SiO}$ in a gas at $1000 \mathrm{~K}$.

Correspondingly, $f_{-}^{(\mathrm{r})}$ and $f_{-}^{(\mathrm{i})}$ are the real and imaginary parts of the profile function at

$v_{-}=\left(\omega-\left[\omega_{0}-g \Omega / 2\right]\right) \frac{1}{\Delta \omega_{\mathrm{D}}}$

and $f_{0}^{(\mathrm{r})}$ and $f_{0}^{(\mathrm{i})}$ at

$v_{0}=\left(\omega-\omega_{0}\right) \frac{1}{\Delta \omega_{\mathrm{D}}}$.

The complex profile function $f(\omega)$ is given by

$f(\omega)=\frac{1}{\pi} \int_{-\infty}^{+\infty} \frac{\Phi(v) \mathrm{d} v}{\Gamma+\mathrm{i}\left(\omega_{0}-\omega\left[1-\frac{v}{c}\right]\right)}$ 
Table 2. Summary of models.

\begin{tabular}{lrrrrrr}
\hline \hline Resonance frequency & $\omega_{\mathrm{R}}$ & $=$ & $2.71 \times 10^{11} \mathrm{~s}^{-1}$ & \\
FWHM of velocity distribution & $\Delta v$ & $=$ & $1.0 \mathrm{~km} \mathrm{~s}^{-1}$ & & \\
Planetary magnetic moment & $M$ & $=$ & $34 \mathrm{G}_{J}^{3}$ & & \\
Tilt of magnetic dipole axis & $\Theta$ & $=$ & $10^{\circ}$ & & & \\
Maser gain length & $L$ & $=$ & $10 r_{J}$ & & & \\
\hline Model & $1 \mathrm{a}$ & $1 \mathrm{~b}$ & $2 \mathrm{a}$ & $2 \mathrm{~b}$ & $2 \mathrm{c}$ & $2 \mathrm{~d}$ \\
Distance of maser slab from rotation axis $\left[r_{J}\right]$ & 3 & 3 & 3 & 3 & 0 & 0.5 \\
Height above equatorial plane $\left[r_{J}\right]$ & 0 & 0 & 0 & 0 & 3 & 3 \\
Diameter of maser bundle $\left[r_{J}\right]$ & 0 & 3 & 0 & 3 & 0 & 0 \\
Normalized peak intensity & 10 & 10 & 0.1 & 0.1 & 0.1 & 0.1 \\
\hline
\end{tabular}

where $\Gamma$ is the decay rate of the excited state $\left(5 \mathrm{~s}^{-1}\right.$ for the $\mathrm{SiO}$ masers considered here, see Nedoluha \& Watson 1994), and $\Phi(v)$ is the velocity distribution of the masing gas, which is taken to be Maxwellian. The velocity distribution of molecules in different substates differs from a pure Maxwellian distribution, because Eqs. (1-3) are velocity-dependent. The real and imaginary parts of $f(\omega)$ are known as the Voigt function $\mathcal{H}$ and the Faraday-Voigt function $\mathcal{F}$, respectively, namely

$f(v, a)=\frac{1}{\sqrt{\pi}}[\mathcal{H}(a, v)+2 \mathrm{i} \mathcal{F}(a, v)]$

with $a=\Gamma / \Delta \omega_{\mathrm{D}}$. For $\mathcal{H}$ and $\mathcal{F}$ I used the rational approximation given by Humlicek (1982), as provided in an optimized form by Schreier (1992).

Without a detailed model at hand, I include neither the magnetopause nor the magnetotail, but assume that the $\mathrm{SiO}$ maser slab crosses a magnetic dipole field (in spherical coordinates, defined by the symmetry axis of the dipole field, e.g., Lewis 2004), given by

$$
\begin{aligned}
B_{r} & =\frac{2 M}{r^{3}} \cos \theta, \\
B_{\theta} & =\frac{M}{r^{3}} \sin \theta, \\
B_{\phi} & =0 \mathrm{G},
\end{aligned}
$$

where $M=34 \mathrm{G} r_{J}^{3}$ is the magnetic dipole moment ( $r_{J}$ is Jupiter's mean radius). The justification of using such a magnetic field component is discussed in Appendix A. We now transform the magnetic dipole field from its stationary frame into a coordinate system defined by the planetary rotation axis, $\boldsymbol{e}_{z}=(0,0,1)^{\mathrm{T}}$, the line-of-sight towards the observer, $\boldsymbol{e}_{x}=(1,0,0)^{\mathrm{T}}$ and $\boldsymbol{e}_{y}=$ $(0,1,0)^{\mathrm{T}}$, such that $\left(\boldsymbol{e}_{x}, \boldsymbol{e}_{y}, \boldsymbol{e}_{z}\right)$ form a left-handed coordinate system (Fig. 1). The transformation is defined by the Euler angles $\Phi=2 \pi t / T=\Omega t$ (where $T$ is the rotation period, and $\Theta$ the inclination of the magnetic dipole axis against the rotation axis), and is obtained by

$$
\left(\begin{array}{l}
x^{\prime} \\
y^{\prime} \\
z^{\prime}
\end{array}\right)=M(t)\left(\begin{array}{l}
x \\
y \\
z
\end{array}\right)
$$

with

$$
M(t)=\left(\begin{array}{rrr}
\cos \Omega t & \sin \Omega t & 0 \\
-\cos \Theta \sin \Omega t & \cos \Theta \cos \Omega t & \sin \Theta \\
\sin \Theta \sin \Omega t & -\sin \Theta \cos \Omega t & \cos \Theta
\end{array}\right)
$$

(in Fig. 1, $\Phi=90^{\circ}$ has been chosen). For $T$ and $\Theta$, values close to those for Jupiter are used, i.e., $T=10 \mathrm{~h}$ and $\Theta=10^{\circ}$, assuming that the planet is still too far away from the star to lose angular momentum to spin up the latter (Soker 2001). Keeping the typical values for giant gas planets in the solar system is therefore not too farfetched in this context.

\section{Results and discussion}

In the following, the model results (Figs. 2 to 7) are shown as grey-scale plots (fractional polarization or polarization angle vs. phase and velocity) and as polarization spectra at a given reference phase of the planetary rotation, or as a time series at a given reference velocity. To search for strong magnetic fields, maser features with a strong fractional circular polarization $\left(p_{\mathrm{C}}\right)$ are needed (which also suffer less than linear polarization from the confusion in the main beam of the spatially unresolved observations). I therefore use the phase and velocity of the maser feature with the strongest $p_{\mathrm{C}}$ as reference.

\subsection{Saturated maser}

The first model 1a (see Table 2 and Fig. 2) is for a maser with a pencil beam in the planet's equatorial plane, at $3 r_{J}$ from the center of the planet, with a length of $10 r_{J}$. The opacity is scaled such that the peak intensity is $10 I_{\mathrm{S}}$, i.e., the maser is saturated. Because of both the small tilt of the magnetic dipole axis against the rotation axis, and the absence of a toroidal magnetic field component $B_{\phi}, \cos \gamma \simeq 0$ and the linear polarization does only slightly exceed the canonical value of $33 \%$ (Goldreich et al. 1973) for a saturated maser propagating perpendicular to the magnetic field. (Our excess stems from line-of-sight elements with $\left.\cos ^{2} \gamma>2 / 3\right)$. The peak-to-peak variation in the fractional linear polarization, $p_{\mathrm{L}}$, is $6.6 \%$ at line center, and $3.4 \%$ at the velocity of maximum circular polarization. The circular polarization displays the archetypical S-shaped line profile of an unresolved Zeeman pattern whose peak-to-peak amplitude varies in time by $2.6 \%$. This effect will be observable provided that the maser is sufficiently strong. The relatively strong circular polarization is not only produced by the Zeeman effect (term $\mathrm{C}$ in Eq. (8)), but also increased by the rotation of the magnetic field in the maser amplification zone along the line-of-sight, rotating the linear polarization from Stokes $Q$ to Stokes $U$ via the E-term in Eq. (8), and then its conversion from Stokes $U$ to Stokes $V$ via the D-term.

In observational practice, the situation is often less clear, for two reasons. First, while polarization monitoring with singledish telescopes offers the advantage of being free of fluctuations due to baseline synthesis effects in VLBI observations, it lacks the spatial resolution of the latter. What is obtained is instead a spectral blend of Zeeman features from individual maser spots, centered at different velocities. If the full width to zero Stokes $V$ 

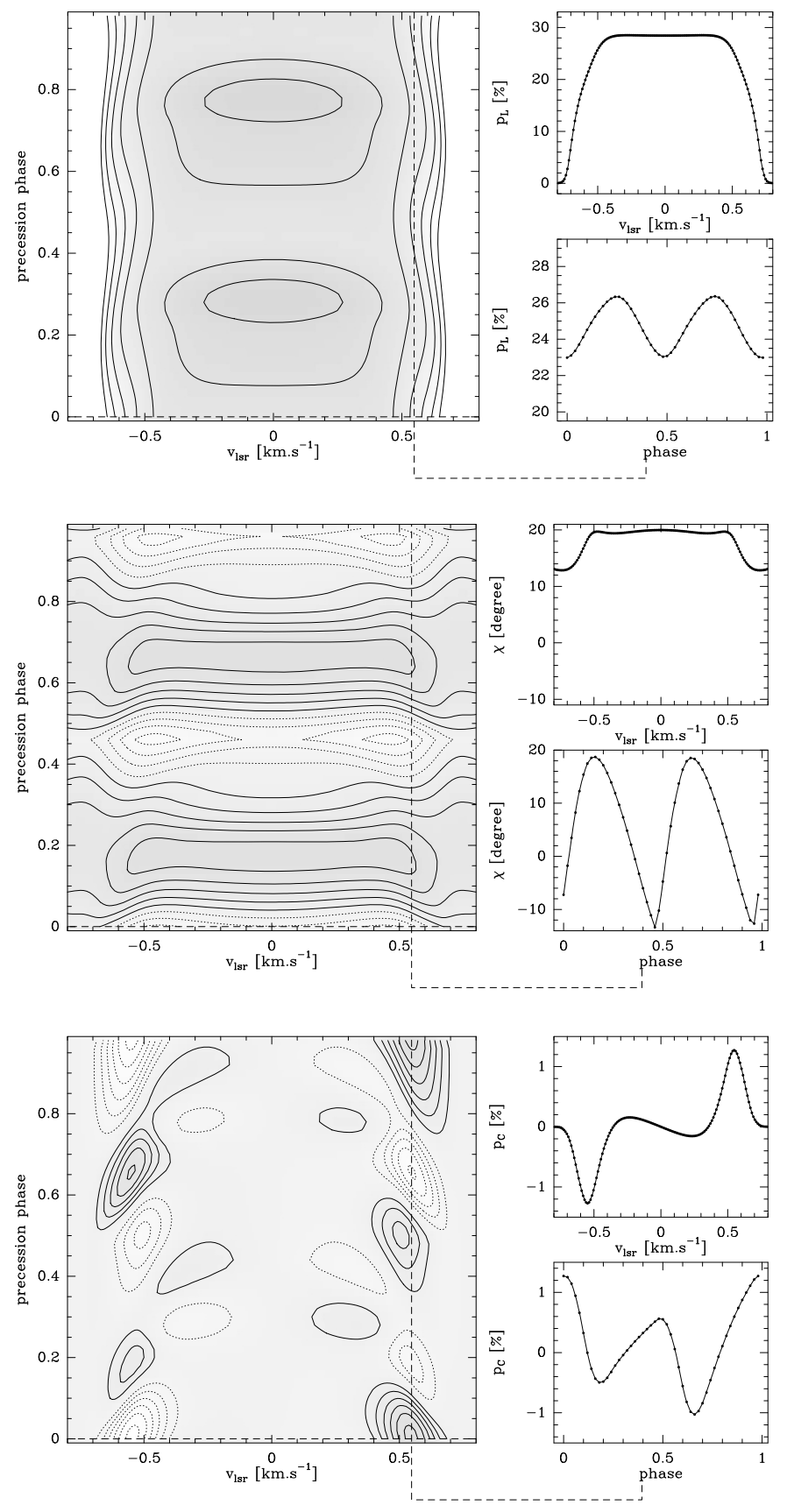

Fig. 2. Saturated $\mathrm{SiO}$ maser $\left(I_{\text {peak }}=10 I_{\mathrm{S}}\right)$, pencil beam at $r=3 r_{J}$, in the planet's equatorial plane (model 1a). Inserts: spectra and time series at the phase (indicated by a horizontal dashed line), respectively velocity (indicated by a vertical dashed line), of maximum circular polarization. Each dot represents a point of the time sampling. Top: fractional linear polarization $\left(p_{\mathrm{L}}\right.$ vs. velocity and phase (contour levels $15 \%$ to $33 \%$ by $3 \%$ ). Center: position angle $\chi$ of linear polarization East from North, contour levels $-18^{\circ}$ to $+18^{\circ}$ by $4^{\circ}$ ). Bottom: fractional circular polarization $\left(p_{\mathrm{C}}\right.$, contour levels from $-1.2 \%$ to $+1,2 \%$ by $0.2 \%$, zero contour suppressed).

from an individual feature is below the velocity separation of two spatially separated maser features, observations with a high enough spectral resolution will help. If not, this results in confusion. Even the spectra of spatially resolved maser spots may be concerned in the case of a velocity gradient along a single maser slab, leading to velocity redistribution. As already stated above, the latter case is not handled here, with the rationale of restricting the models to the basic cases before dealing with the complications (which, in the limit of a uni-directional slab maser, can of course be handled by an extension of the theory described above). Nature may help us here, because the magnetic flux density in a planetary magnetosphere may be enhanced with respect to that of a magnetic field of stellar origin, but this hope needs to be ascertained. We cannot expect that all detections of a strong Stokes $V$ in $\mathrm{SiO}$ masers (corresponding to a few up to $20 \mathrm{G}$, Herpin et al. 2006) are owing to planetary magnetospheres.

The second caveat concerns both VLBI and single-dish observations. In reality, $\mathrm{SiO}$ masers in Mira stars have a typical size of at least $\sim 10^{12} \mathrm{~cm}$ (constrained both from VLBI observations, e.g., Philips \& Boboltz 2000, and models, Bujarrabal 1994), which is much larger than the length scale on which the magnetic dipole field varies in model 1a. To simulate the effects of diluting the magnetosphere in the maser cross section, a cylindrical maser slab (model variant $1 \mathrm{~b}$ ) has also been modeled as a collection of maser rays with a bundle diameter of $3 r_{J}$ (i.e., two orders of magnitude below observed spot sizes). This corresponds, at a distance of e.g., $230 \mathrm{pc}$, to an angular size of 0.01 mas (i.e., still unresolved by VLBI observations). Assuming a larger and more realistic size would require including the magnetopause and the magnetotail in the model, and possibly the detailed dynamics of mass accretion onto the planet, which clearly is beyond the scope of this paper. I therefore assume that the polarization signals modeled here are on top of a linear or circular polarization background, which does not vary on a timescale comparable to that of the planetary rotation. We know that the lifetime of $\mathrm{SiO}$ masers is unlikely to be longer than a cycle of the stellar variability, because the features are disrupted by shock waves driven by the stellar pulsation (Humphreys et al. 2002). Multiepoch VLBA imaging of $\mathrm{SiO} v=1, J=1-0$ masers impressively confirmed this picture (the "movie" of TXCam, Diamond \& Kemball 2003). Long-term polarization fluctuations may occur on shorter timescales (several weeks at least, Glenn et al. 2003). They come from slow readjustments of the magnetic field. These timescales are long enough to monitor at least 10 periods of the planetary rotation, allowing us to separate the short-term polarization fluctuations modeled here from the longterm ones of polarized and total maser flux.

The polarization background is not necessarily dominant, even in single-dish observations. Linear polarization is reduced by the combined action of both the predominantly tangential polarization of the maser spots distributed along a ring (providing indirect evidence of radiative pumping, Desmurs et al. 1999) and cancellation of polarization vectors in the observing beam. As for circular polarization, we may have reasons to expect that it is enhanced towards the lines of sight crossing the strongest magnetic fields, i.e., close to the planet, as suggested above. Any observational attempt to discover the features modeled here will therefore focus on measurements of the circular polarization. Figure 3 shows that the maximum linear polarization is reduced by about $5.3 \%$ due to dilution (even in VLBI observations, since they do not resolve the maser spot either), and incoherent mixing correspondingly reduces the peak-to-peak variation of the polarization angle. As expected, the circular polarization basically remains the same. (Because of the mixing of maser rays with smaller Zeeman splittings, the velocity of maximum $p_{\mathrm{C}}$ is reduced.) At the velocities of maximum circular polarization, the relative peak-to-peak variation of the linear polarization has doubled with respect to the pencil-beam model (1a), thanks to the shift in the velocity of maximum circular polarization (which is used as reference). 

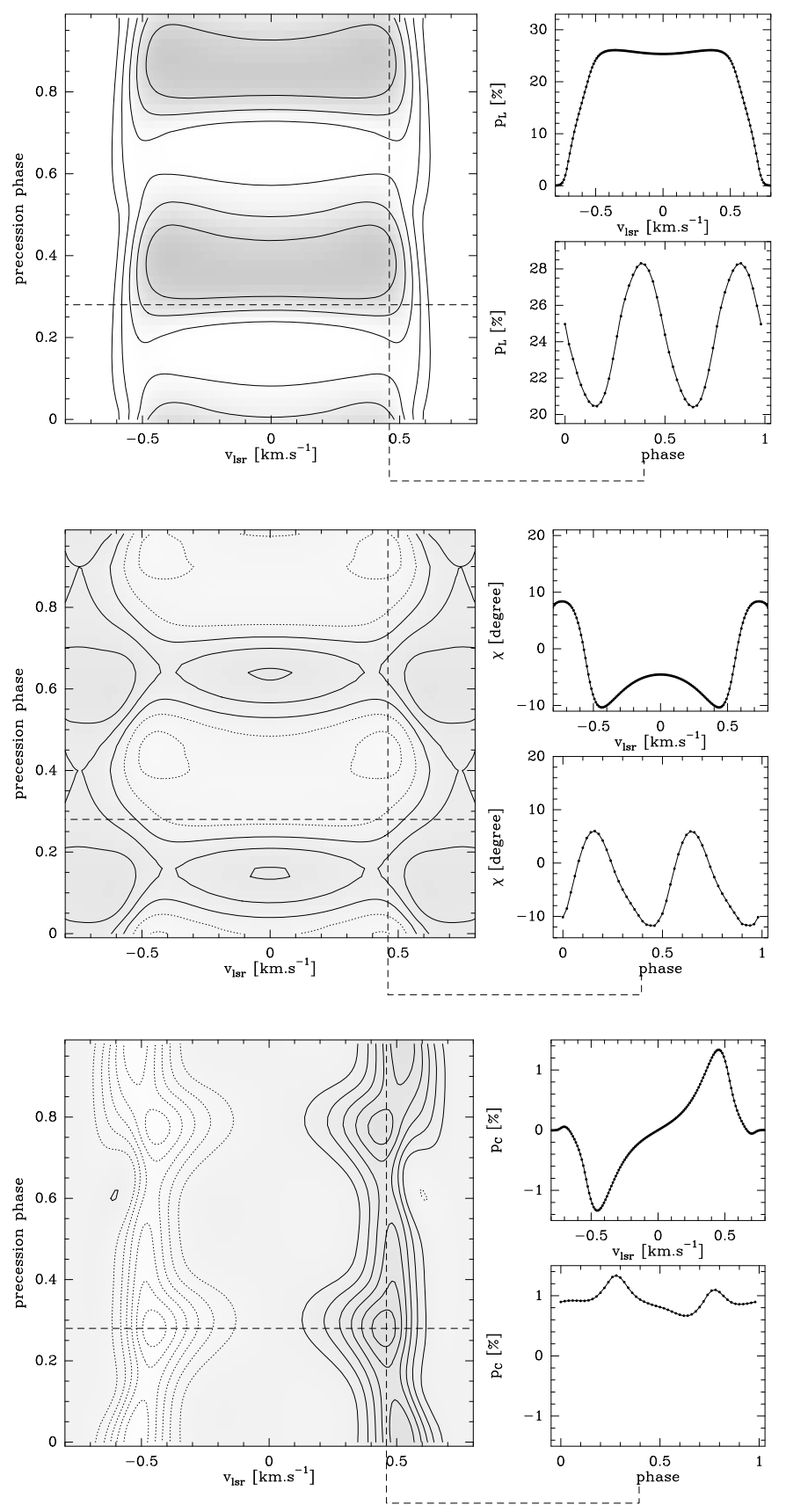

Fig. 3. Same as Fig. 2, but for a bundle of $\mathrm{SiO}$ masers, with a slab diameter of $3 r_{J}$ (model $1 \mathrm{~b}$ ). For easy comparison, the plot limits for the inserts are the same as those for the equivalent pencil beam model (Fig. 2). Top: $p_{\mathrm{L}}$, contour levels from $15 \%$ to $27 \%$ by $3 \%$. Center: $\chi$, contour levels from $-10^{\circ}$ to $+10^{\circ}$ by $5^{\circ}$. Bottom: $p_{\mathrm{C}}$, contour levels from $-1.2 \%$ to $+1.2 \%$ by $0.2 \%$ (zero contour omitted).

\subsection{Unsaturated maser}

The saturation of masers is difficult to determine experimentally, mainly due to its dependence on the observationally poorly constrained beaming angle (Watson \& Wyld 2001). The polarization of unsaturated masers is weaker than that of their saturated counterparts, because only the latter convert each pumping event into a maser photon. A fully unsaturated maser $(\Gamma \ll R)$ is unpolarized if both the seed and pump radiation are unpolarized. The model 2a (Table 2 and Fig. 4) is for a pencil beam with $0.1 I_{\mathrm{S}}$, and yields a maximum linear polarization of $4.4 \%$ at line center (with a peak-to-peak variation of $1.3 \%$ ), which drops to $2.6 \%$ at the velocity of strongest circular polarization (with a peak-topeak amplitude of $0.8 \%$ ). As expected in unsaturated masers, the line profile of Stokes $V$ is identical to that of thermal radiation (Fiebig \& Güsten 1989, see also Watson \& Wyld 2001), and the linewidths are narrowed with respect to the thermal width (cf. Watson \& Wyld, Fig. 2). Therefore, the circular polarization is drastically reduced (to $p_{\mathrm{C}}= \pm 0.009 \%$ ) for a maser ray in the equatorial plane, and is no longer detectable with current sensitivity. Again, a cylindrical maser slab (model 2b, Fig. 5) does not drastically alter the result for circular polarization, but reduces the linear polarization at the reference channel to a maximum of $1.5 \%$, with a peak-to-peak variation of $0.3 \%$ (i.e., mean value and amplitude are scaled down by a factor 1:2).

For the detectability of the circular polarization of an unsaturated $\mathrm{SiO}$ maser from a Jovian magnetosphere, its location in the latter is important. Because the circular polarization here is proportional to the line-of-sight component of the magnetic field (hereafter $B_{\text {los }}$ ), it is naturally enhanced for maser rays crossing the rotation axis, which have a maximum $B_{\text {los }}$ before and after the crossing. This is demonstrated by model (2c) in Fig. 6, where the maser ray is otherwise at the same distance from the planet as in model (2a). The peak-to-peak variation of $p_{C}= \pm 0.29 \%$ now becomes detectable with a carefully tuned polarimeter (whereas the maximum linear polarization drops to $p_{\mathrm{L}}=1.5 \%$ ).

For lines of sight close to the rotation axis, $B_{\text {sky }}$ changes strongly as a function of both velocity and rotation phase. Model $2 \mathrm{~d}$ (Fig. 7) is for a pencil beam $3 r_{J}$ above the equatorial plane, and slightly offset $\left(0.5 r_{J}\right)$ from the rotation axis. The dependency of the polarization angle on both $B_{\text {los }}$ and the magnetic field component projected onto the plane of the sky, $B_{\text {sky }}$ leads to a complicated pattern. The polarization angle flips by $90^{\circ}$ are because of the critical $\cos ^{2} \gamma=1 / 3$, which decides whether the linear polarization is along $B_{\text {sky }}$ or perpendicular to it (Goldreich et al. 1973), and of the variable maser saturation across the line profile (hence the strong spectral variations). Observationally, these flips have been proven by VLBI polarization maps (i.e., Kemball \& Diamond 1997) and hint at variations of the magnetic field either due to a planetary magnetic field as modeled here or a magnetic field component of stellar origin.

\subsection{The impact of refinements of the radiative model}

\subsubsection{Anisotropic pumping}

$\mathrm{SiO}$ masers are believed to be radiatively pumped by infrared excitation of vibrationally excited states (Gray et al. 2009, further references therein). The anisotropic distribution of the pump radiation leads to an unequal population of the magnetic sublevels and therefore enhances the linear polarization (Elitzur 1996), which generates a circular polarization in excess of its value for isotropic pump rates if magnetorotation is present (as in the models presented here). This birefringent conversion from linear to circular polarization would make a detection of fluctuations of $p_{\mathrm{C}}$ possible even in the unsaturated regime. The same presumably holds for anisotropic loss rates. In the case of anisotropic pumping, the critical value of $\gamma$ for producing linear polarization parallel or perpendicular to $B_{\text {sky }}$ will not be the $55^{\circ}$ of Goldreich et al., though the linear polarization will still have one of these orientations with respect to the magnetic field. 

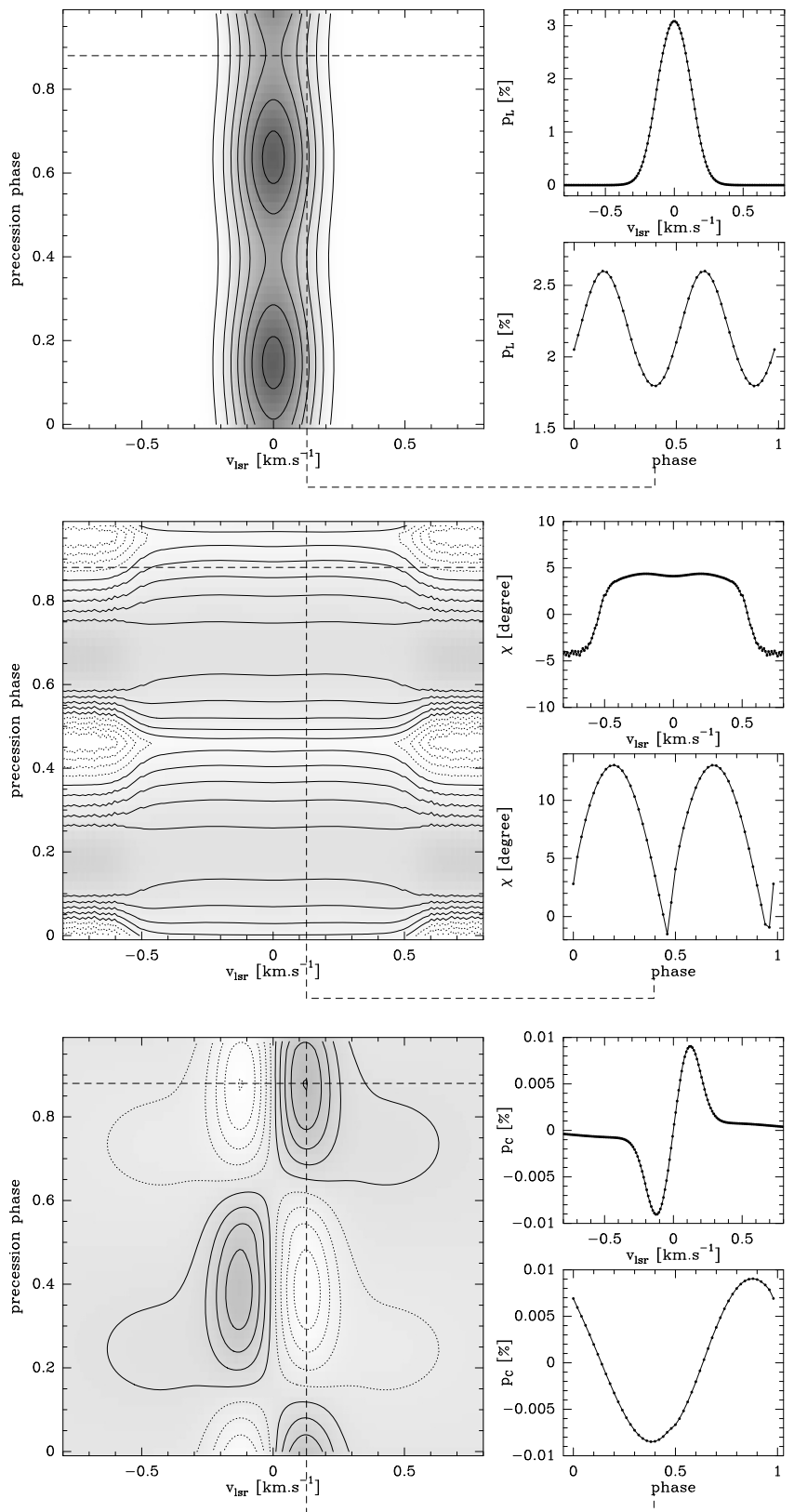

Fig. 4. Unsaturated $\mathrm{SiO}$ maser $\left(I_{\text {peak }}=0.1 I_{\mathrm{S}}\right)$, pencil beam at $r=3 r_{J}$, in the planet's equatorial plane (model $2 \mathrm{a}$ ). The linewidths are narrowed with respect to the thermal width, as expected for unsaturated maser action. Top: $p_{\mathrm{L}}$, contour levels from $0.6 \%$ to $4.2 \%$ by $0.6 \%$. Center: $\chi$, contour levels $-12^{\circ}$ to $12^{\circ}$ by $3^{\circ}$. Bottom: $p_{\mathrm{C}}$, contour levels from $-0.009 \%$ to $+0.009 \%$ by $0.002 \%$.

\subsubsection{More complex maser geometries}

A refined model would imply not only a more adequate description of the magnetosphere crossed by the $\mathrm{SiO}$ maser, but also a maser geometry more complicated than the uni-directional cylindrical maser slabs considered here. The first step to a more realistic description of the maser action is to consider bidirectional masers (i.e., two opposed propagation directions in a linear maser). For a constant magnetic field, Watson \& Wyld (2001) show that the circular polarization remains nearly unaltered between uni- and bi-directional masers, whereas the linear polarization has a tendency to be increased in the last (while qualitatively keeping its dependency on maser saturation and $\cos \gamma)$.

\subsubsection{Higher rotational quantum numbers}

The comparison between the $v=1, J=2-1$ maser transition and its $v=1, J=1-0$ counterpart (both taken to be unidirectional) shows that their circular polarization is not drastically different. As for linear polarization, Deguchi \& Watson (1990) found that the polarization of the $J=2-1$ transition is actually suppressed with respect to the 1-0 transition when $(g \Omega)^{2} / \Gamma \gg R \gg g \Omega$, while observationally the former tends to be enhanced (McIntosh \& Predmore 1993), such that strong linear polarizations can only be achieved by other than magnetic means (e.g., the aforementioned anisotropic pumping, as proposed, in this context, by Watson \& Wyld 2001). However, this regime does not allow us to analyze the magnetic field geometry by means of polarization measurements, because a single Larmor precession of the molecule is destroyed by a stimulated emission before its completion. I therefore did not consider this case here.

\subsection{The impact of instrumental limitations}

\subsubsection{Spatial resolution}

While the quasi-stationary linear polarization tends to decrease due to mutual cancellation within the beam of spatially unresolved observations, the variations in the circular polarization from different maser spots would need to fluctuate in anticorrelation in order to cancel. This is highly unlikely, even if all variations were caused by the effect discussed here, and even more if other phenomena - e.g., jets or coronal flux loops, occurring on different timescales - were involved. These scenarios are discussed in Wiesemeyer et al. (2009). The observational results of that work, and subsequent polarization monitoring from December 2008, indicate that fluctuations of the linear and circular polarizations may appear simultaneously (as shown here) or separately (in contrast to the models presented in this paper). The possibility of a blend of several unrelated phenomena calls for a coordinated observational effort as proposed in our 2009 paper. The direct imaging of planetary wake flows in the atmospheres of the nearest AGB stars with ALMA will definitely be an asset. According to Cherchneff (2006), we can expect suitable molecular high-density tracers to be sufficiently abundant in the atmosphere, thanks to the non-equilibrium chemistry dominated by shock propagation.

\subsubsection{Spectral resolution}

The Stokes spectra shown in Figs. 2 to 7 are calculated with a frequency resolution of $2 \pi \Delta v=10 \mathrm{kHz}$, corresponding to a velocity resolution of $0.01 \mathrm{~km} \mathrm{~s}^{-1}$, which in practice cannot be achieved with existing correlation polarimeters. After smoothing with a box-like kernel, 12 frequency steps wide, the circular peak polarization of model 1a (Fig. 2) drops from $\pm 1.27 \%$ to $\pm 0.94 \%$, which is still observable (i.e., the spectral smoothing does not lead to an order-of-magnitude loss of circular polarization). The decrease in circular polarization due to the limited spectral resolution depends on the width of the Zeeman feature. A comparison between the $p_{\mathrm{C}}$ spectra in Figs. 2 to 7 shows that the relative decrease of the circularly polarized signal remains comparable for the unsaturated masers. However, here the weakness of the polarization will make a detection difficult, if not 

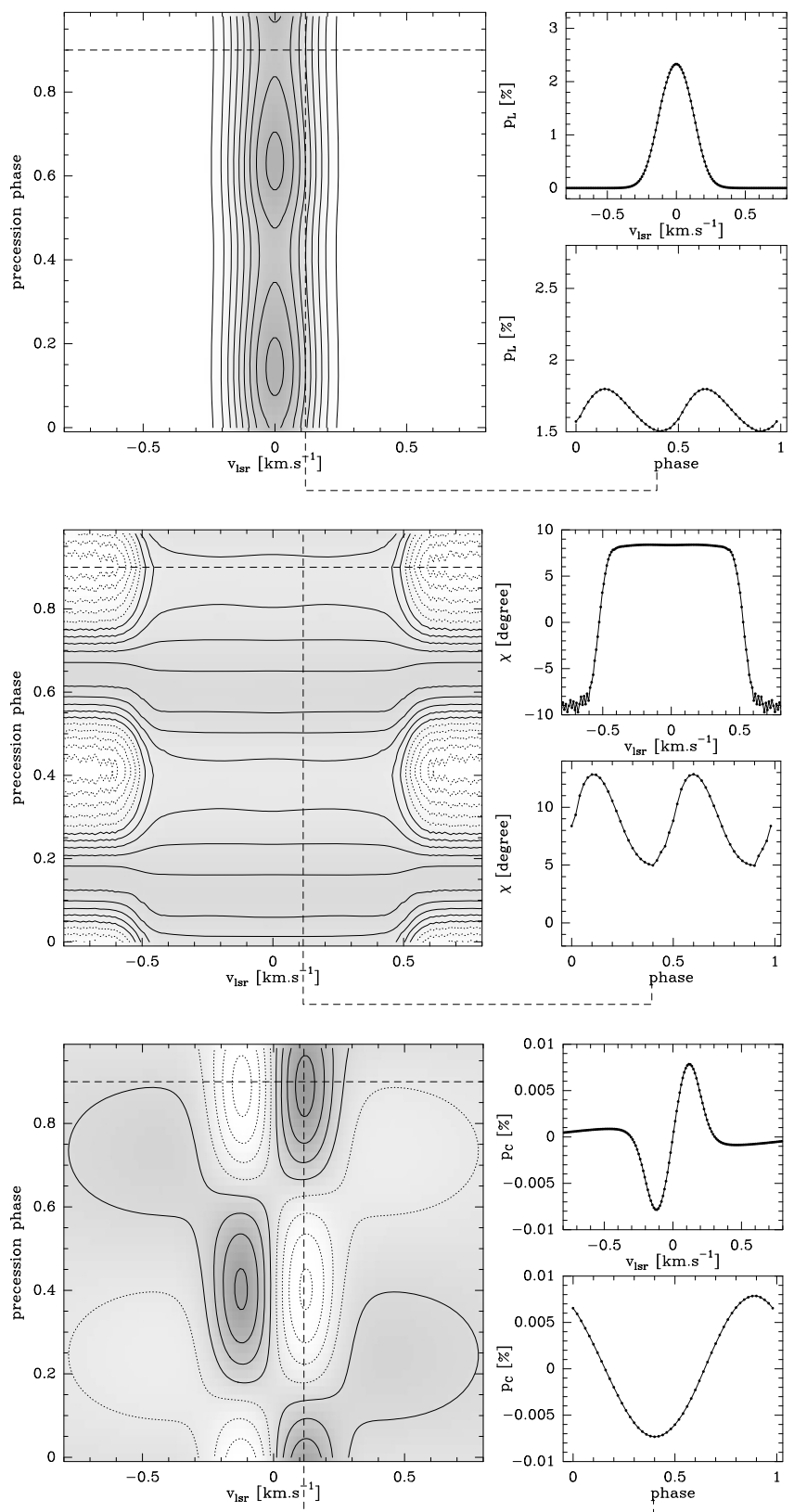

Fig. 5. Same as Fig. 4, but for a bundle of $\mathrm{SiO}$ masers, with slab diameter of $3 r_{J}$ (model $2 \mathrm{~b}$ ). Top: $p_{\mathrm{L}}$, contour levels $0.3 \%$ to $2.7 \%$ by $0.3 \%$. Center: $\chi$, contour levels from $-15^{\circ}$ to $+12^{\circ}$ by $3^{\circ}$. The "noise" in the spectrum of $\chi$ is a numerical artifact. Bottom: $p_{\mathrm{C}}$, contour levels from $-0.007 \%$ to $+0.007 \%$ by $0.002 \%$.

impossible. The polarization monitoring therefore has to be done with the best possible spectral resolution.

\section{Conclusions}

I have shown that densely sampled polarization monitoring is able to reveal precessing Jovian magnetospheres engulfed in the atmospheres of AGB stars by a periodic modulation of the polarization characteristics of $\mathrm{SiO}$ masers crossing them. The expected period is that of the rotation of giant gas planets (i.e., $\sim 10 \mathrm{~h}$ if the rotation period did not change with respect to typical solar system values), which allows us to distinguish such a fingerprint from long-term variations of the polarized (Glenn et al. 2003) and unpolarized maser flux
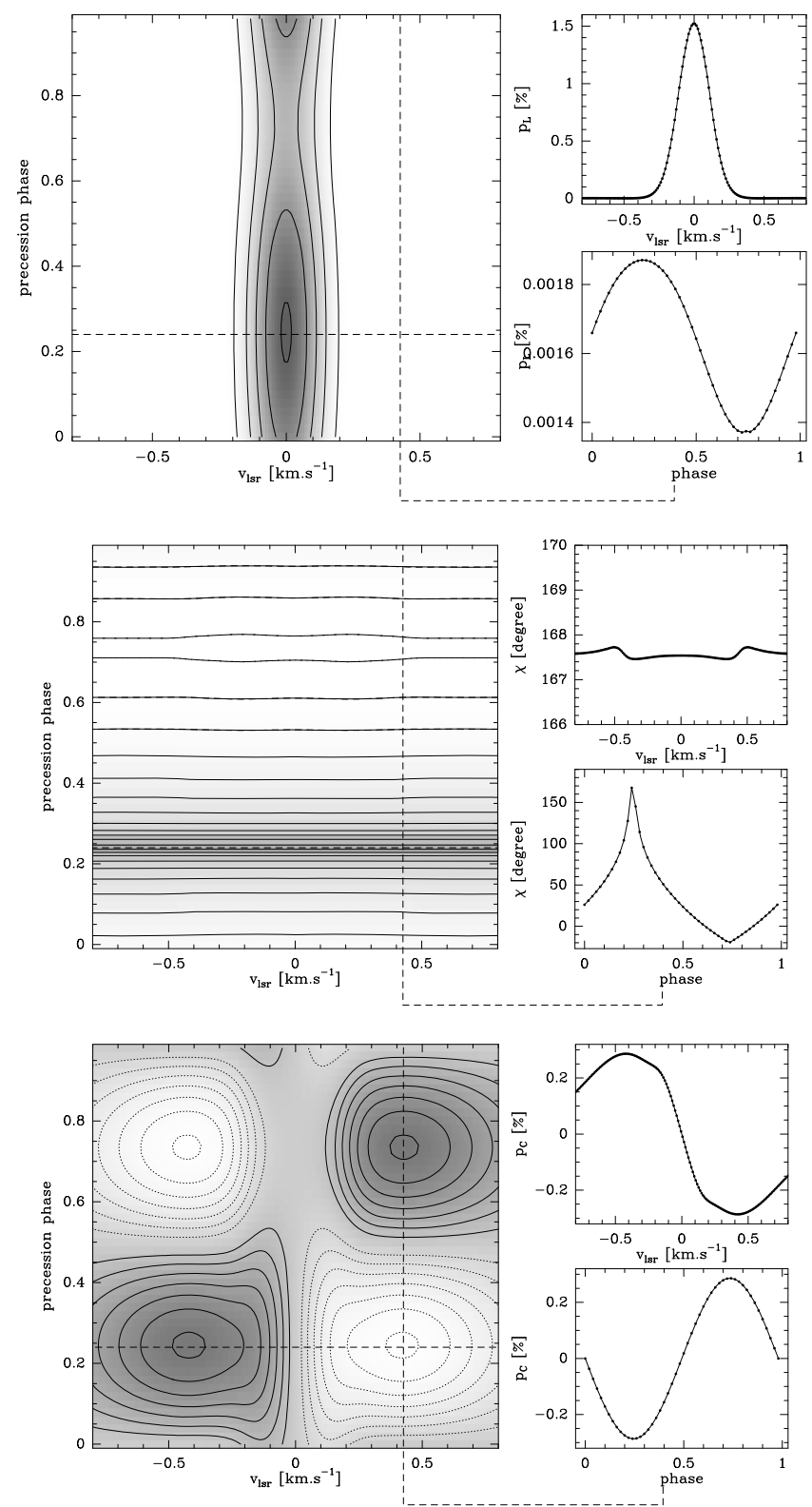

Fig. 6. Same as Fig. 4, but $3 r_{J}$ above the planet's equatorial plane and towards its rotation axis (model 2c). Top: $p_{\mathrm{L}}$, contour levels $0.3 \%$ to $1.5 \%$ by $0.3 \%$. Center: $\chi$, contour levels from $-16^{\circ}$ to $+160^{\circ}$ by $16^{\circ}$. Bottom: $p_{\mathrm{C}}$, contour levels from $-0.28 \%$ to $+0.28 \%$ by $0.04 \%$.

(Humphreys et al. 2002; Diamond \& Kemball 2003), which occur on timescales at least an order of magnitude longer than those considered here. As for the distinction from pseudoperiodic polarization variations due to stellar magnetic flux loops or magnetic clouds, everything depends on the speed of the $\mathrm{SiO}$ masers relative to the latter. At a speed of $10 \mathrm{~km} \mathrm{~s}^{-1}$, a maser covers a distance of $5 r_{J}$ within $10 \mathrm{~h}$, i.e., the patterns modeled here are only visible if the maser speed across the planetary magnetosphere is $<10 \mathrm{~km} \mathrm{~s}^{-1}$, or if the precession period is $<10 \mathrm{~h}$. Otherwise, polarization fluctuations are expected that not only contain temporal variations, but also spatial ones, and the patterns will be either compressed or stretched. A planetary magnetosphere exposed to an AGB wind has its magnetopause closer to the planet than e.g., Jupiter's magnetopause, which is exposed to the faster, but less dense solar wind. As a matter of fact, the case of a planetary magnetosphere in an AGB wind may be closer 

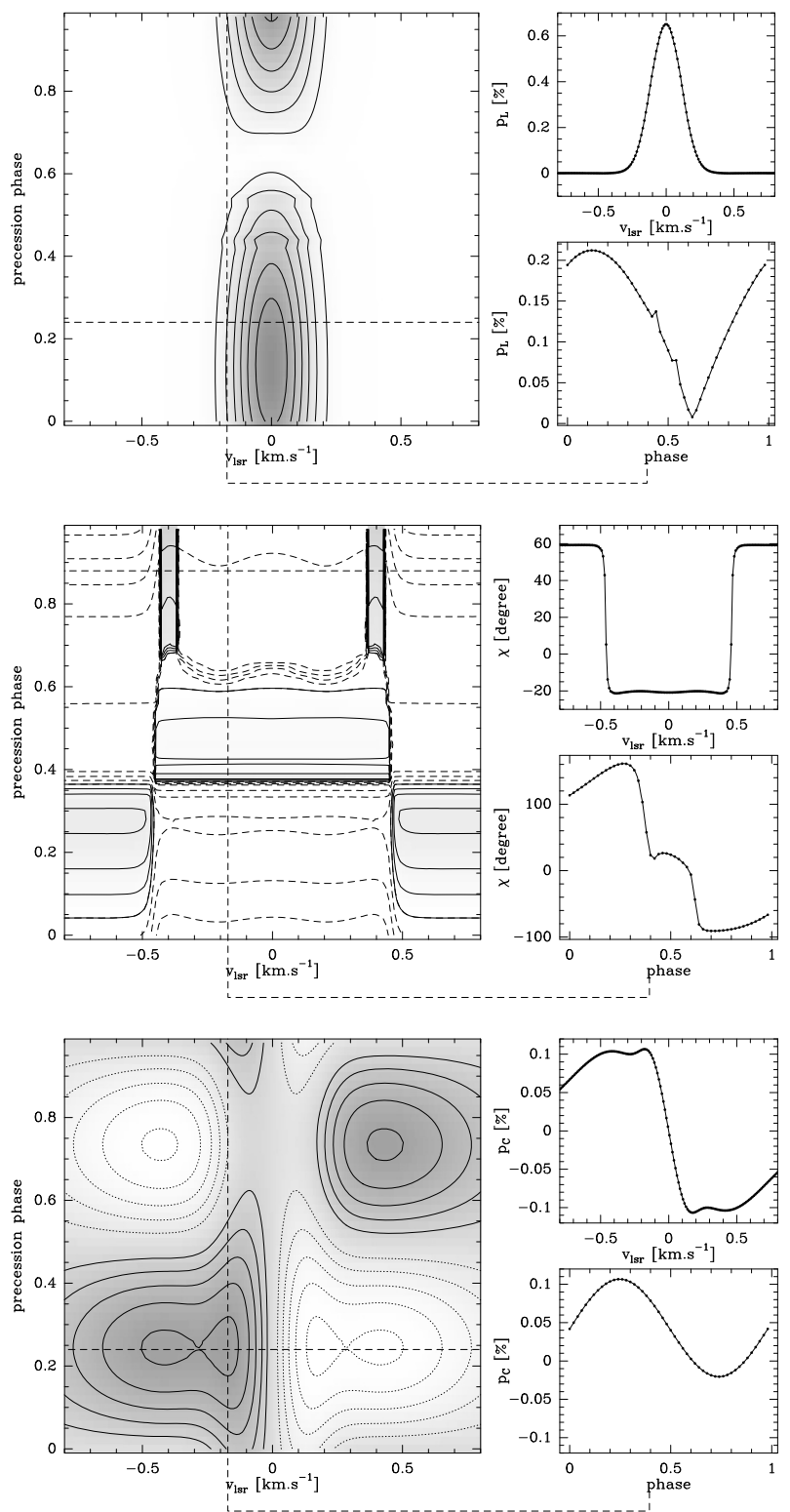

Fig. 7. Same as Fig. 6, but slightly offset from the rotation axis (by $0.5 r_{J}$, model $2 \mathrm{~d}$ ). Top: $p_{\mathrm{L}}$, contour levels $0.1 \%$ to $0.6 \%$ by $0.1 \%$ (the small jumps in the insert showing the time series of $p_{\mathrm{L}}$ are a numerical artifact). Center: $\chi$, contour levels from $-16^{\circ}$ to $+160^{\circ}$ by $16^{\circ}$. Bottom: $p_{\mathrm{C}}$, contour levels from $0.02 \%$ to $+0.1 \%$ by $0.02 \%$.

to that of the terrestial magnetosphere, whose magnetopause is as close as a few Earth radii, and can even be temporarily disrupted in case of strong solar storms. Likewise, the magnetic dipole component of a planetary magnetic field engulfed by the atmosphere of an AGB star can be expected to be quite compact (not more than 10 planetary radii). To observe the modeled features, an $\mathrm{SiO}$ maser has to cross it, and either needs to be saturated to produce a measurable circular polarization, be unsaturated if the pump and loss rates are sufficiently anisotropic, or has to cross a part of the magnetosphere with a strong lineof-sight component of the magnetic field. This leads to the conclusion that the phenomenon will be rare and, if detected, only be seen in a narrow range of velocities, and preferentially in circular polarization-enhancing regions of strong magnetic flux density (whereas linear polarization tends to cancel out if the polarization angle strongly varies across the observing beam).
Furthermore, the dynamics of the gas close to the planet possibly accreting matter needs to be such that the line-of-sight velocity coherence allows for the maser action. The abundance of $\mathrm{SiO}$ molecules required to form a strong enough maser can be expected to be less of a problem. The evaporation of Galilean moons has been proposed as an additional reservoir for the gasphase $\mathrm{SiO}$ (Struck-Marcell 1988).

Recently, Wiesemeyer et al. (2009) have detected a pseudoperiodic variation of the fractional circular polarization towards two Mira stars, R Leo, and V Cam, which may hint at precessing magnetospheres in their atmospheres. Several periods will have to be monitored, to better distinguish the phenomenon from other rapid, but transient magnetospheric events. The case of $\mathrm{R}$ Leo is especially intriguing, since VLBA maps of the $43 \mathrm{GHz}$ $\mathrm{SiO}$ maser (Cotton et al. 2004, 2008; Soria-Ruiz et al. 2007) show elongated $\mathrm{SiO}$ features pointing radially away from the star, reminiscent of jet-like features. The velocity information from the observed polarization fluctuations, together with the astrometry of such elongated features, suggest orbital motion in at least one of them, and strengthens the case for a planetary wake flow as simulated by Struck et al. (2004). Dense polarization monitoring of these features may be especially rewarding, and a spatially fully resolved polarization monitoring will certainly be highly conclusive. However, VLBI techniques will suffer from a confusion between polarized flux variations and variations due to Earth rotation synthesis of visibility components (the timescale for both is unfortunately the same). This calls for a combination of single-dish polarization monitoring with VLBI imaging of the Stokes $I$ emission. The cross-identification of polarization fluctuations with VLBI features is possible if there is, in the single-dish observations, no blend of spatially separated $\mathrm{SiO}$ maser spots at the same velocity or if the VLBI features show an unambiguous association with a planetary wake flow. Detection of orbital motion in such features will provide a touchstone for the scenarios presented here. The aim of this paper has been to motivate coordinated observational efforts, so far unprecedented, and to show that meaningful results can be expected to emerge from them. If so, this will call for advanced modeling. The work published here should therefore be considered as the initial step in an iterative refinement.

Acknowledgements. This work has been improved by the valuable comments of C. Thum and W. D. Watson, who also helped to make the text more readable and to avoid misunderstandings. I acknowledge the constructive comments of an anonymous referee and the assistance of the computer group of IRAM Granada in providing the computing power for the numerical calculations. I would like to thank J. Adams and C. Halliday for their careful language editing of this and a related paper.

\section{Appendix A: The magnetosphere model}

Before modeling a Jovian magnetosphere exposed to an AGB wind, it has to be shown whether it can resist the latter, i.e., whether it is able to form a magnetopause far enough from the planet to allow for a protected magnetic dipole field, such that the ram pressure of the inflowing wind equals the magnetic pressure of the magnetosphere (Chapman-Ferraro boundary, see e.g., Kennel 1995). This is the case if the magnetic flux density in the magnetopause, $B_{\mathrm{mp}}$, is

$B_{\mathrm{mp}}=0.05\left(\frac{\rho_{\mathrm{W}}}{10^{-16} \mathrm{~g} \mathrm{~cm}^{-3}}\right)^{1 / 2}\left(\frac{v_{\mathrm{W}}}{10 \mathrm{~km} \mathrm{~s}^{-1}}\right) \mathrm{G}$,

where $\rho_{\mathrm{W}}$ and $v_{\mathrm{W}}$ are the density and velocity of the wind, respectively (Struck-Marcell 1988), scaled to the typical values for 
an AGB wind. The assumed Jovian magnetic dipole field reaches this value in the equatorial plane at a distance

$r_{\mathrm{mp}}=\left(\frac{M}{B_{\mathrm{mp}}}\right)^{\frac{1}{3}}$.

For a magnetic moment of $M[\mathrm{G}]=34 r_{J}^{3}$ (eight times the Jovian value), a dipole magnetosphere can extend to up to 8.8 Jovian radii (substellar distance from the planet). For a weaker magnetic field, the magnetopause would be closer to the planet, but it would still be possible to have an $\mathrm{SiO}$ maser crossing the precessing magnetosphere in an extended magnetotail.

A comparison with the solar system may be instructive here. The Jovian magnetopause extends to $92 r_{J}$, which is about double what would be expected from Eq. (A.1) for solar system parameters $\left(n_{\mathrm{W}}=0.14 \mathrm{~cm}^{-3}, v_{\mathrm{W}}=400 \mathrm{~km} \mathrm{~s}^{-1}\right)$, showing that the Jovian magnetosphere is more complex than a magnetic dipole field protected by a magnetopause (Alexeev \& Belenkaya 2005). Only a strong enough interplanetary magnetic field can push the magnetopause beyond this limit. In the atmosphere of an AGB star, even more complex phenomena may be expected, because of the high density of the wind and the possibility of a reconnection between the planetary and stellar magnetic field. For comparison, the terrestial subsolar magnetopause may be as close at $6 r_{\mathrm{E}}$ to the Earth's center, depending on space weather (Shue et al. 1997), i.e., at a distance (relative to the Earth's radius) rather similar to that of the Jovian model planet (relative to $r_{J}$ ) than to that of Jupiter. Likewise, the location of a planetary magnetopause in an AGB wind depends on the variations in the latter, especially in response to the stellar pulsations. These timescales are, however, far longer than those considered here $(\sim 10 \mathrm{~h})$. To avoid a perturbation of a precessing magnetosphere by the AGB wind and the accretion of matter onto the planet, the magnetic flux needs to be decoupled from the matter via ambipolar diffusion. As for the latter, I estimate its speed $v_{\text {ad }}$ following Hartquist \& Dyson (1997), but using values closer to the magnetosphere modeled here, by

$$
\begin{aligned}
v_{\mathrm{ad}} \simeq 1000 \mathrm{~km} \mathrm{~s}^{-1} & \left(\frac{B}{1 \mathrm{G}}\right)^{2}\left(\frac{L}{0.1 r_{J}}\right)^{-1}\left(\frac{X_{i}}{10^{-6}}\right)^{-1} \\
& \times\left(\frac{n_{\mathrm{H}}}{10^{10} \mathrm{~cm}^{-3}}\right)^{-2}
\end{aligned}
$$

where $L \simeq 0.1 r_{J}$ is the typical length scale on which the modeled magnetic field varies, $B \simeq 1 \mathrm{G}$ is the typical magnetic flux density in the model magnetosphere, $X_{\mathrm{i}} \simeq 10^{-6}$ the ionization fraction, and $n_{\mathrm{H}} \simeq 10^{10} \mathrm{~cm}^{-3}$ the expected hydrogen density in the maser slab. The major uncertainty in Eq. (A.3) comes from the gas density (especially if shocks are involved) and the ionization fraction. In any realistic case, the suggested ambipolar diffusion speed of $\sim 1000 \mathrm{~km} \mathrm{~s}^{-1}$ is much faster than the precession speed of the magnetosphere $\left(2 \pi r_{J} / T \sim 12 \mathrm{~km} \mathrm{~s}^{-1}\right.$ with a period of $T=10 \mathrm{~h}$ ), and the magnetic flux and matter can decouple efficiently, such that the magnetic field lines diffuse through the maser slab. This conclusion holds for the localized magnetic field considered here. As for the global magnetic field pervading the AGB atmosphere, Soker (2006) rules out any dynamical importance, because of the weak coupling between the magnetic flux and the matter, based on a similar argument. I conclude that a planetary magnetic dipole field can persist in the extended atmosphere of an AGB star. Ohmic dissipation of the magnetic flux can be neglected here, otherwise the substantial circular polarizations of $\mathrm{SiO}$ masers could not have been observed.

\section{Appendix B: Numerical calculations}

Because the growth rates of the Stokes parameters in the propagation of maser photons differ by orders of magnitude at different velocities, Eq. (7) is a stiff set of differential equations, and numerical solutions using Runge-Kutta or multiple-order methods are inefficient, even with adaptive step sizes. Because the Stokes parameters at different frequencies are not independent of each other due to the Zeeman splitting, the calculations have to be done along the full zero-to-zero width of the spectral line, with a sampling dense enough to resolve the Zeeman feature. For a given time step $t$, the calculations proceed along the lineof-sight in steps that are short enough to solve the equation of radiative transfer using the matrix exponential function $\exp (\boldsymbol{K} \tau)$ with piecewise constant coefficients, i.e., for a step from grid point $j$ to $j+1$

$\boldsymbol{I}_{j+1}=\exp (\boldsymbol{K} \tau) \boldsymbol{I}_{j}$

where the elements of the vector $\boldsymbol{I}_{j}$ are the Stokes parameters entering the grid cell. Spontaneous emission is neglected, as usual in maser theory. Landi degl'Innocenti \& Landi degl'Innocenti (1981) have provided an elegant analytical solution for the matrix exponential function in closed form. At each sampling point along the line-of-sight, we calculate the magnetic field from Eqs. (19)-(23), the corresponding angles $\gamma$ and $\eta$ from Eq. (6), the resulting Zeeman splitting $g \Omega$, and the corresponding matrix elements $A$ to $E$. For a given line-of-sight element and frequency, the stimulated emission rates in Eqs. (1) to (5) are determined from the normalized Stokes intensities entering it by a Spline interpolation of the spectral line profiles in $(I, Q, U, V)^{\mathrm{T}}$. I assume a linear, unidirectional maser, with unpolarized continuum seed radiation. For each rotation phase considered, results are shown for both a maser spot much smaller than the length scale on which the magnetic field typically varies, and for a maser spot consisting of a number of independent, parallel rays (hereafter called bundle). As already mentioned, for distances farther than $8 r_{J}$ away from the planet the model would not be valid anymore, and would have to be modified by the shocked AGB wind, the magnetopause, and the magnetotail. Without a detailed magnetohydrodynamical model at hand, we assume that all masers of the bundle are centered on an impact parameter of $3 r_{J}$ (such that the maser is confined within the magnetic dipole field), have the same line-of-sight velocity, and are characterized by a total gain length of $10 r_{J}$. The tidal interaction of the masing gas with the possibly matter accreting planet leads to a loss of velocity coherence and thus of maser saturation. I therefore model the effects of an incoherent velocity field by comparing unsaturated maser action with a saturated one. For the case of a homogeneous magnetic field the code has been successfully tested by comparing its results whith those of Watson \& Wyld (2001).

\section{References}

Alexeev, I. I., \& Belenkaya, E. S. 2005, Annales Geophysicae, 23, 809 Asensio Ramos, A., Landi Degl'Innocenti, E., \& Trujillo Bueno, J. 2005, ApJ, 625,985

Barvainis, R., McIntosh, G., \& Predmore, C. R. 1987, Nature, 329, 613 Bujarrabal, V. 1994, A\&A, 285, 971

Bujarrabal, V., \& Nguyen-Q-Rieu 1981, A\&A, 102, 65

Cherchneff, I. 2006, A\&A, 456, 1001

Cotton, W. D., Mennesson, B., Diamond, P. J., et al. 2004, A\&A, 414, 275

Cotton, W. D., Perrin, G., \& Lopez, B. 2008, A\&A, 477, 853

Deguchi, S., \& Watson, W. D. 1986, ApJ, 300, L15 
Deguchi, S., \& Watson, W. D. 1990, ApJ, 354, 649

Desmurs, J.-F., Bujarrabal, V., Colomer, F., \& Alcolea, J. 1999, New Astron. Rev., 43, 559

Diamond, P. J., \& Kemball, A. J. 2003, ApJ, 599, 1372

Elitzur, M. 1991, ApJ, 370, 407

Elitzur, M. 1996, ApJ, 457, 415

Fiebig, D., \& Guesten, R. 1989, A\&A, 214, 333

Glenn, J., Jewell, P. R., Fourre, R., \& Miaja, L. 2003, ApJ, 588, 478

Goldreich, P., Keeley, D. A., \& Kwan, J. Y. 1973, ApJ, 179, 111

Gray, M. D. 2003, MNRAS, 343, L33

Gray, M. D., Wittkowski, M., Scholz, M., et al. 2009, MNRAS, 394, 51

Hartquist, T. W., \& Dyson, J. E. 1997, A\&A, 319, 589

Herpin, F., Baudry, A., Thum, C., Morris, D., \& Wiesemeyer, H. 2006, A\&A, 450, 667

Herwig, F. 2005, ARA\&A, 43, 435

Humlícek, J. 1982, J. Quant. Spectr. Rad. Trans., 27, 437

Humphreys, E. M. L., Gray, M. D., Yates, J. A., et al. 2002, A\&A, 386, 256

Kemball, A. J., \& Diamond, P. J. 1997, ApJ, 481, L111

Kennel, C. F. 1995, Convection and Substorms: Paradigms of Magnetospheric Phenomenology, published by (US: Oxford University Press)

Landi Deglinnocenti, E., \& Landi Deglinnocenti, M. 1981, Nuovo Cimento B Serie, 62, 1

Lewis, J. S. 2004, Physics and Chemistry of the solar system (Academic Press), 218

McIntosh, G. C., \& Predmore, C. R. 1993, ApJ, 404, L71
Nedoluha, G. E., \& Watson, W. D. 1994, ApJ, 423, 394

Niedzielski, A., Konacki, M., Wolszczan, A., et al. 2007, ApJ, 669, 1354

Phillips, R. B., \& Boboltz, D. A. 2000, AJ, 119, 3015

Santos, N. C. 2008, New Astron. Rev., 52, 154

Sargent, M., Lamb, W. E., \& Fork, R. L. 1967, Phys. Rev., 164, 436

Schreier, F. 1992, J. Quant. Spectr. Rad. Trans., 48, 743

Setiawan, J., Henning, T., Launhardt, R., et al. 2008, Nature, 451, 38

Shue, J.-H., Chao, J. K., Fu, H. C., et al. 1997, J. Geophys. Res., 102, 9497

Silvotti, R., Schuh, S., Janulis, R., et al. 2007, Nature, 449, 189

Soker, N. 2001, MNRAS, 324, 699

Soker, N. 2006, PASP, 118, 260

Soker, N. 1996, ApJ, 460, L53

Soria-Ruiz, R., Alcolea, J., Colomer, F., Bujarrabal, V., \& Desmurs, J.-F. 2007, A\&A, 468, L1

Struck, C., Cohanim, B. E., \& Willson, L. A. 2002, ApJ, 572, L83

Struck, C., Cohanim, B. E., \& Willson, L. A. 2004, MNRAS, 347, 173

Struck-Marcell, C. 1988, ApJ, 330, 986

Thum, C., Wiesemeyer, H., Paubert, G., Navarro, S., \& Morris, D. 2008, PASP, 120, 777

Villaver, E., \& Livio, M. 2007, ApJ, 661, 1192

Wang, Z., Chakrabarty, D., \& Kaplan, D. L. 2006, Nature, 440, 772

Watson, W. D., \& Wyld, H. W. 2001, ApJ, 558, L55

Western, L. R., \& Watson, W. D. 1984, ApJ, 285, 158

Wiesemeyer, H. W., Thum, C., Baudry, A., \& Herpin, F. 2009, A\&A, 498, 801

Wolszczan, A., \& Frail, D. A. 1992, Nature, 355 